\title{
Analysis of in vitro Glucose Utilization in a Circadian Pacemaker Model
}

\author{
George C. Newman, ${ }^{1,2}$ Frank E. Hospod,, ${ }^{1,2}$ Clifford S. Patlak, ${ }^{1}$ and Robert Y. Moore ${ }^{3}$ \\ Departments of Neurology and Neurological Surgery, State University of New York at Stony Brook, Stony Brook, \\ New York 11794, 2VA Medical Center, Northport, New York 11768, and the 32Departments of Psychiatry, \\ Neurology, and Behavioral Neuroscience and the Center for Neurosciences, University of Pittsburgh, \\ Pittsburgh, Pennsylvania 15261
}

\begin{abstract}
An in vitro glucose utilization method, based upon ${ }^{14} \mathrm{C}-2-$ deoxyglucose kinetics in brain slices, has been used to study circadian rhythms in hypothalamic slices containing the suprachiasmatic nucleus (SCN). Spontaneous SCN metabolic activity in vitro is similar to that observed in vivo with higher metabolic rates in subjective daytime and lower rates during subjective night. However, in vitro SCN metabolic activity during late subjective day is above that seen when glucose utilization is measured in vivo, suggesting that an inhibitory influence normally active in vivo is lost during slice isolation. Incubation of slices containing SCN in the presence of TTX exposes a TTX-insensitive component of metabolic activity in early subjective day, supporting prior suggestions that glucose utilization by the circadian oscillator continues in the absence of $\mathrm{Na}^{+}$-dependent action potentials. Studies with high $\mathbf{M g}^{2+}$ concentrations are consistent with the hypothesis that most metabolic activity above the basal level observed with the glucose utilization method is related to synaptic activity. Pharmacological studies of the SCN brain slice model with radiotracers offer potential for analysis of both circadian rhythmicity and neural regulation.
\end{abstract}

The suprachiasmatic nucleus ( $\mathrm{SCN}$ ) of hypothalamus is the principal circadian pacemaker in mammals, driving a wide variety of behavioral and physiological rhythms (Moore and Eichler, 1972; Stephan and Zucker, 1972). Despite detailed knowledge about SCN anatomy and physiology (Meijer and Rietveld, 1989), the cellular mechanisms involved in circadian pacemaker function remain largely unknown. Intrinsic circadian rhythms demonstrated in SCN in vivo thus far include a rhythm of neuronal action potential firing rate, which persists even when the nucleus is isolated as a surgical "island" (Inouye and Kawamura, 1979), a rhythm of glucose utilization demonstrated with the ${ }^{14} \mathrm{C}-2$ deoxyglucose (2DG) method (Schwartz et al., 1980), and a rhythm of vasopressin (VP) synthesis (Schwartz and Reppert, 1985; Reppert and Uhl, 1987; Robinson et al., 1988). SCN glucose utilization is high in daytime and low at night. Recently it has

\footnotetext{
Received Sept. 16, 1991; revised Dec. 17, 1991; accepted Dec. 23, 1991.

This research is supported by a VA Merit Review Grant and Biomedical Research Support Grant NIH-05376, funds from the Department of Neurology, and NIH Grant NS-16304. We thank Ms. Priscilla Wu for her valued technical assistance and Drs. Janet and Finbarr O'Sullivan for their assistance with the statistical analyses.

Correspondence should be addressed to Dr. George C. Newman, Department of Neurology, HSC T12-020, SUNY at Stony Brook, Stony Brook, NY 11794

Copyright (C) 1992 Society for Neuroscience $0270-6474 / 92 / 122015-07 \$ 05.00 / 0$
}

been shown that hypothalamic infusion of TTX in vivo appears to block SCN input and output pathways without affecting the circadian oscillator itself (Schwartz et al., 1987a), suggesting that neural firing is a pacemaker output rather than an intrinsic component of oscillator function. This also implies that there may be components of the rhythm of glucose utilization related to intrinsic oscillator function that may be exposed by eliminating the large metabolic costs of neuronal electrical activity with TTX. In order to address this and other aspects of SCN neurochemistry, we have developed an in vitro model of SCN utilizing hypothalamic brain slices and 2DG autoradiography. The unique circadian properties of the SCN, combined with the advantages of in vitro pharmacology, can also help to define the cellular processes that contribute to glucose utilization measurements.

Circadian rhythms of neural firing have been repeatedly observed in brain slices (Green and Gillette, 1982; Groos and Hendriks, 1982; Shibata et al., 1982; Wheal and Thomson, 1984), indicating that oscillator function continues in vitro. Initial hypothalamic slice experiments demonstrated that SCN uptake of 2DG is high in subjective daytime and low in subjective night, even after $8 \mathrm{hr}$ in vitro (Newman and Hospod, 1986). Subsequently, we have developed a detailed kinetic model to permit quantification of in vitro glucose utilization so that the glucose consumption related to individual cellular activities might be separated (Newman et al., 1990). In this article, we describe the circadian cycle of SCN glucose utilization in brain slices. We also show, for the first time, that a rhythm of glucose utilization persists in the presence of TTX and describe the effects of $\mathrm{Mg}^{2+}$ on daytime $\mathrm{SCN}$ glucose utilization in vitro.

\section{Materials and Methods}

Materials. All buffers and inorganic salts were obtained from Sigma Chemical (St. Louis, MO) and were cell culture grade. The $95 \% \mathrm{O}_{2}, 5 \%$ $\mathrm{CO}_{2}$ mixture and liquid nitrogen were purchased from General Welding (Long Island City, NY). All ${ }^{14} \mathrm{C}$-2-deoxyglucose (specific activity, 59 $\mathrm{mCi} / \mathrm{mmol}$ ) and ${ }^{14} \mathrm{C}$ standards were from Amersham (Arlington Heights, IL). Isopentane, class IA, was obtained from Fisher Scientific (Fair Lawn, NJ), and scintillation cocktail, from National Diagnostics (Manville, NJ). OM-1 film for autoradiography was obtained from Kodak (Rochester, NY). All water was deionized and purified to a resistance of $17.5 \mathrm{M} \Omega$ with a Barnstead NANOpure system (Boston, MA). Sprague--Dawley rats were obtained from Taconic Farms (Germantown, NY).

Tissue preparation and incubations. Brain slice incubations are conducted in a chamber spccifically designed for biochemistry and morphology. Details of our methods for tissue preparation, 2DG incubation, freezing, cryostat sectioning, image analysis, and calculation of glucose 
utilization have been published recently (Newman et al., 1989, 1990), and therefore our methods are discussed only briefly here. Male Sprague-Dawley rats weighing 175-225 gm are caged unrestrained in groups of three at $22^{\circ} \mathrm{C}$ with free access to food and water in a $12 \mathrm{hr}$ : $12 \mathrm{hr}$ light/dark (L/D) cycle for at least 3 weeks. The three cagemates are killed on a single day and divided among control and experimental groups so that a time-matched control accompanies most tetrodotoxintreated preparations. All animals are killed under dim red lighting and the eyes are covered with black electrical tape prior to turning on room lights for brain removal. For animals killed during subjective daytime, the lights are not turned on the day of death so that they receive no light stimulus for at least $12 \mathrm{hr}$ prior to death. Animals killed during the dark phase of the L/D cycle are handled similarly but without alteration of their lighting schedule prior to death. The brain is removed by a posterior approach to avoid traction on the optic nerve. The hypothalamus is block dissected, and slices are chopped coronally at 540 $\mu \mathrm{m}$ on a Smith-Farquhar tissue chopper (Sorvall, Newtown, CT). Two slices containing the entire SCN are placed in a chamber within 4 min of animal death for preincubation in Krebs-Ringer (K-R) with an atmosphere of prehumidified $95 \% \mathrm{O}_{2}, 5 \% \mathrm{CO}_{2}$ and $10 \mathrm{~mm}$ glucose, 1.5 mM Ca ${ }^{2+}$ pH $7.37,305$ mOsm and $\mathrm{PO}_{2}$ of $715 \mathrm{~mm} \mathrm{Hg}$. Slices are preincubated for $75 \mathrm{~min}$ prior to isotope exposure. Slices incubated with TTX or the various $\mathrm{Mg}^{2+}$ concentrations are exposed to the altered buffer for 15 min prior to incubation with $2 D G$, during the $2 \mathrm{DG}$ incubation, and throughout the rinse period.

Measurement of glucose utilization. Measurement of in vitro glucose utilization with $2 \mathrm{DG}$ is analogous to in vivo glucose utilization (Sokoloff et al., 1977) except that it is possible to deliver a square wave pulse of radioactivity to the tissue. Incubation with $2 D G$ is initiated by moving the slices to a second chamber preequilibrated with K-R containing 0.2 $\mu \mathrm{Ci} / \mathrm{ml}$ of isotope. The slices are incubated for $45 \mathrm{~min}$, removed from the incubation chamber, briefly rinsed in warm $\mathrm{K}-\mathrm{R}$, and returned to the preincubation chamber for $30 \mathrm{~min}$ of rinse. Following rinse, the slices are rapidly frozen in isopentane cooled to $-80^{\circ} \mathrm{C}$ and cryostat sectioned to $20 \mu \mathrm{m}$. Sections are collected on a glass slide, exposed to $\mathrm{x}$-ray film along with standards for 1 week, and then stained with cresyl violet for image analysis. Optical density is measured with an image analyzer (MCID, St. Catherine, Ontario, Canada) that permits image overlay so that the region of interest is chosen while viewing only the Nissl section. Alternate sections are analyzed throughout the rostrocaudal extent of the nucleus except for sections within $80 \mu \mathrm{m}$ of the slice surface that are excluded (Newman, 1991). Radioactivity is quantified in $\mathrm{nCi} / \mathrm{gm}$ tissue using the standard curve. Glucose utilization is then calculated in right and left SCN and the average of right and left anterior hypothalamic areas (AHA) using the measured radioactivity and Equation 1 , which has been derived using a five-compartment, eight-parameter kinetic model (Newman et al., 1990). The mean of all sections from each animal is then calculated for each right and left $\mathrm{SCN}$ and the combined AHA:

$$
\frac{R_{i}}{C_{p}}=\frac{K_{1}{ }^{*} k_{3}{ }^{*}}{\mathrm{LC}\left(k_{2}{ }^{*}+k_{3}{ }^{*}\right.} \cdot \frac{C_{i}{ }^{*} / C_{p}{ }^{*}}{\Sigma(E(i)+B(i)+M(i)+G(i))\left(e^{\lambda_{i} T_{0}}-1\right) e^{\lambda_{i \Delta}}} .
$$

In Equation $1, R_{i}$ is rate of glucose utilization; $C_{p}$ is the concentration of glucose in the perifusate; $C_{i}^{*}$ is the total radioactivity in the tissue; $C_{p}{ }^{*}$ is the perifusate radioactivity; LC is the lumped constant; $K_{1}{ }^{*}, k_{2}{ }^{*}$, and $k_{3}{ }^{*}$ are rate constants; $E(i), B(i), M(i), G(i)$, and $\lambda_{i}$ are kinetic parameters derived from the solution of the differential equations for the model; $T_{0}$ is the time of incubation ( $45 \mathrm{~min}$ ); and $\Delta$ is the duration of rinse $(30 \mathrm{~min})$. Although this equation appears complex, in practice, once the rate constants have been determined for the tissue and $C_{p}, T_{0}$, and $\Delta$ are set, the entire equation reduces to a single constant that is multiplied by $C_{i}^{*} / C_{p}{ }^{*}$ to obtain glucose utilization. For hypothalamic slices incubated under the conditions stated above, Equation 1 reduces to $R_{i}=104.19 \times C_{i}^{*} / C_{p}{ }^{*}$. The circadian time assigned to each slice incubation corresponds to the midpoint of the $45 \mathrm{~min}$ incubation with 2DG.

Analysis. Each time point for slices with or without TTX was performed with 3-12 replicates. All SCN and AHA values are means with standard errors. Comparisons between control and TTX time points and between SCN and AHA were performed by $t$ tests with correction for multiple comparisons by the Bonferroni technique in all cases. Levels of significance are reported for two-tailed $t$ tests in all cases. Individual slices were assigned an exact circadian time (CT) according to the midpoint of the incubation with 2DG. Slices were then combined into CT groups using the following limits (with circadian time, CT00, defined as the time of lights on): CT01, CT00-01.5; CT03, CT01.5-04; CT06, CT04-07.5; CT09, CT07.5-10; CT12, CT10-13; CT15, CT13-16; CT18, CT16-19; CT21, CT19-22; CT23, CT22-24. The same limits were applied for control or TTX-treated slices and were used throughout the text and figures.

Analysis of rostrocaudal profile. In order to study the rostrocaudal profiles of SCN glucose utilization, three control slices, one each from CT06, СT09, and CT12, were chosen for serial section analysis based upon uniform sectioning and tissue chopping that isolated most of the SCN within a single brain slice. Values for right and left SCN were averaged and values for sections within $80 \mu \mathrm{m}$ of the slice surface were corrected for edge artifacts (Newman, 1991). Cross-sectional areas of each SCN half-nucleus was determined using the MCID image analyzer. The three slices were aligned for comparison by using the most rostral section with a unilateral cross-sectional $\mathrm{SCN}$ area of $0.1 \mathrm{~mm}^{2}$ as a reference. Differences in dorsomedial and ventrolateral glucose utilization were also sought using $\mathrm{SCN}$ regions defined with reference to slices that were prepared and incubated in the usual manner and then immunostained with antibodies to VP or vasoactive intestinal peptide.

\section{Results}

\section{Spontaneous in vitro metabolic activity}

Analysis of the means of right and left SCN glucose utilization values for hundreds of slices in this and other experiments has revealed no consistent difference between the two nuclei at any time of day; therefore, we present all SCN data as the average of the right and left nuclei. A robust rhythm of SCN glucose utilization persists in vitro (Figs. 1, 2A). SCN glucose utilization is low from CT 18 to CT23, rises in early subjective day, peaks in late day at CT09, and gradually returns to low levels between CT12 and CT15. Although inspection of the AHA data suggests that there is no diurnal rhythm in that region (Fig. 3.4), comparison between all daytime (CTO0-CT12) and dark-phase (CT12-CT24) control slices reaches significance at the $p<0.05$ level. Most of the in vitro SCN and AHA glucose utilization values are about $20 \%$ higher than in vivo values at the corresponding time of day. SCN values in late subjective day and early dark phase are much higher, however. Individual slice glucose utilization values for nighttime SCN, and for AHA at all times of day, are about $50 \mu \mathrm{mol} / 100 \mathrm{gm} / \mathrm{min}$, while daytime SCN values are considerably higher with several particularly active slices in late day. Variation of SCN glucose utilization values during subjective daytime is considerably greater than variations of SCN values at night or of AHA values at any time of day, similar to the situation observed for neural firing rates in vitro (Shibata et al., 1982; Gillette, 1991).

\section{Rostrocaudal profile}

Rostrocaudal analyses of slices incubated with 2DG at CT06, CT09, and CT12 reveal that in vitro metabolic activity is significantly higher in caudal SCN than in rostral SCN (Fig. 4). Glucose utilization correlates closely with cross-sectional area in the rostral half of the SCN but not at all in the caudal half where values of over $200 \mu \mathrm{mol} / 100 \mathrm{gm} / \mathrm{min}$ are found. It is thus apparent that the high levels of glucose utilization seen during the later half of subjective daytime reflect primarily increased metabolic activity in the caudal half of the nucleus. No consistent differences were observed between the dorsomedial and ventrolateral SCN subdivisions.

\section{Effects of tetrodotoxin on metabolic activity}

Incubation of hypothalamic slices in the presence of $1 \mu_{\mathrm{M}} \mathrm{TTX}$ reduces SCN glucose utilization at CT06, CT09, and CT12 but 

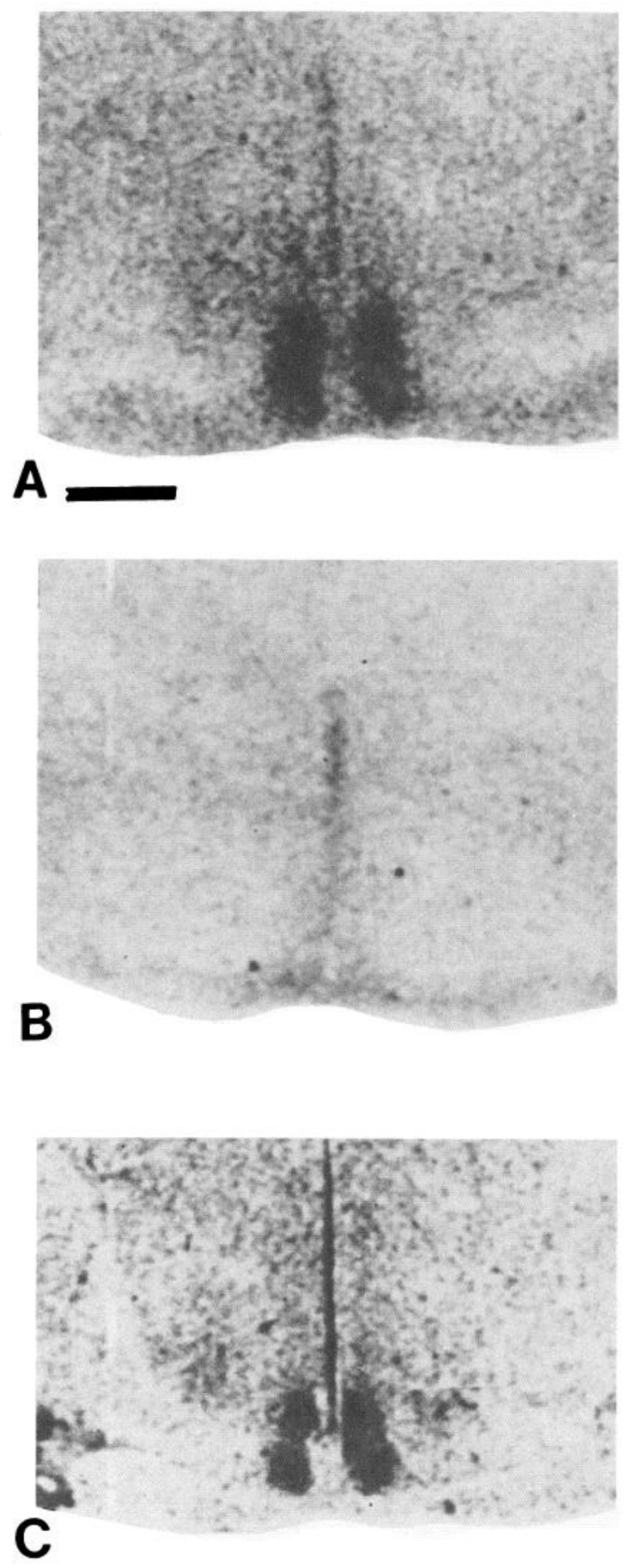

Figure 1. Autoradiograms of $\mathrm{SCN}$ from hypothalamic slices incubated with ${ }^{14} \mathrm{C}-2 \mathrm{DG}$ for in vitro glucose utilization at CT09 (top) or CT21 (center) along with Nissl stain of slice from CT09 (bottom) illustrating the rostrocaudal level from which both autoradiograms are taken. Circadian time (CT) refers to the entraining cycle of the rat, with lights on at CT00 and off at CT12 defining subjective day and dark phase. Scale bar, $1 \mathrm{~mm}$.

does not alter glucose utilization of slices incubated at CT03 or between CT18 and CT23 (Fig. 2B). For all daytime groups except CT03, comparison of control and TTX slices reveals highly significant differences; however, at $\mathrm{CT} 03$ there is no significant decrease produced by TTX. Although there is also no difference between control and TTX-treated slice SCN glucose
A

\section{SCN Controls}

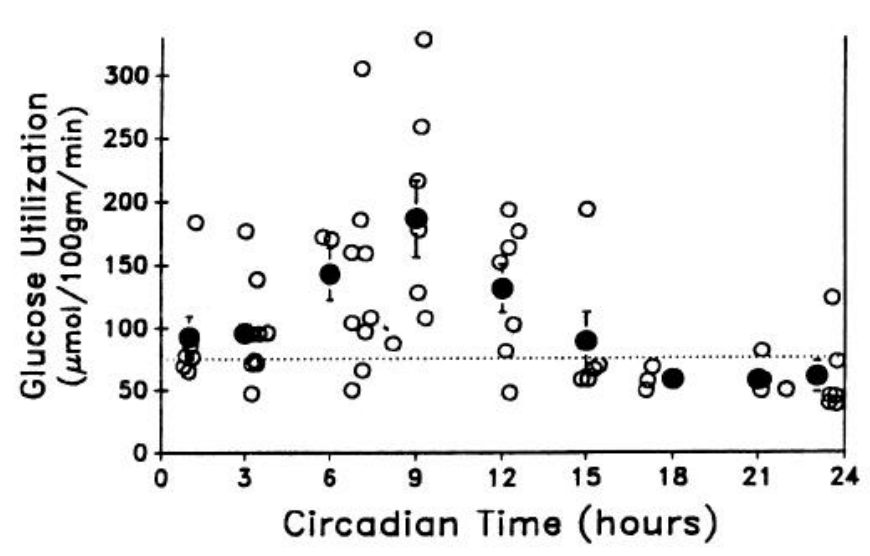

B
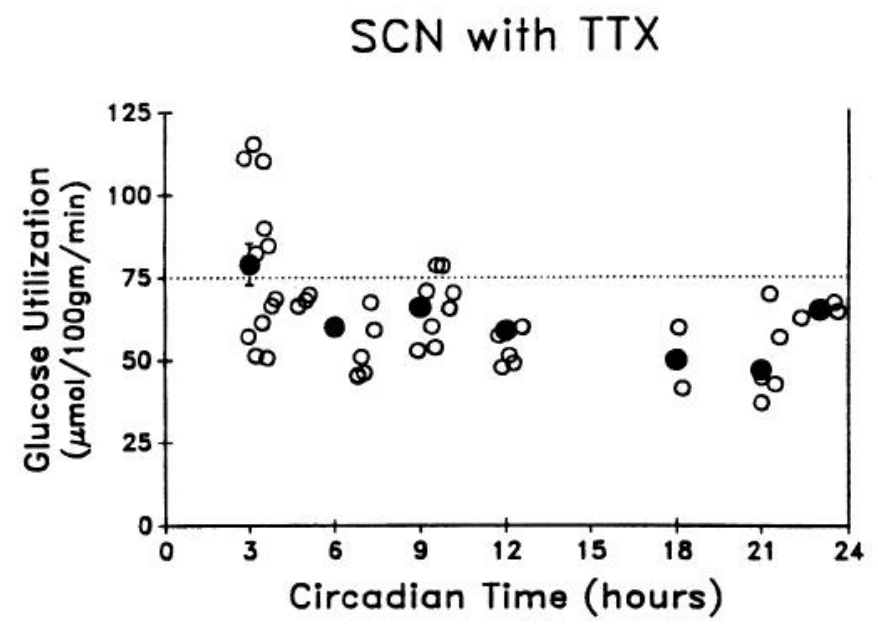

Figure 2. A, SCN glucose utilization of slices incubated under control conditions. $B, \mathrm{SCN}$ glucose utilization of slices incubated in the presence of TTX. Open circles denote individual values of $\mathrm{SCN}$ glucose utilization at exact circadian times, and solid circles refer to means and SEs of slices grouped by circadian times. The horizontal dotted lines at $75 \mu \mathrm{mol} /$ $100 \mathrm{gm} / \mathrm{min}$ are included to facilitate comparison among the graphs which differ in the scale of their y-axis.

utilization during the dark phase of the L/D cycle, control SCN glucose utilization between CT18 and CT21 is low, similar to AHA glucose utilization, whereas SCN glucose utilization of control slices at CT03 is considerably elevated compared to nighttime SCN values or AHA glucose utilization. Glucose utilization of slices incubated with TTX at CT03 also differs significantly from that of slices exposed to TTX between CT06 and CT12 $(p<0.001)$, while slices incubated between CT06 and CT12 do not differ significantly from slices incubated with TTX at CT18 and CT21. Thus, there is persistent spontaneous metabolic activity in $\mathrm{SCN}$ at $\mathrm{CT} 03$ that continues even in the presence of TTX, consistent with the hypothesis that voltageregulated $\mathrm{Na}^{+}$-dependent action potentials are not essential for circadian oscillator function (Schwartz et al., 1987a).

TTX uniformly reduces daytime AHA glucose utilization by $30 \%$ from a mean of $51.2 \pm 12.8$ to $35.5 \pm 6.2$ (Fig. $3 B$; 


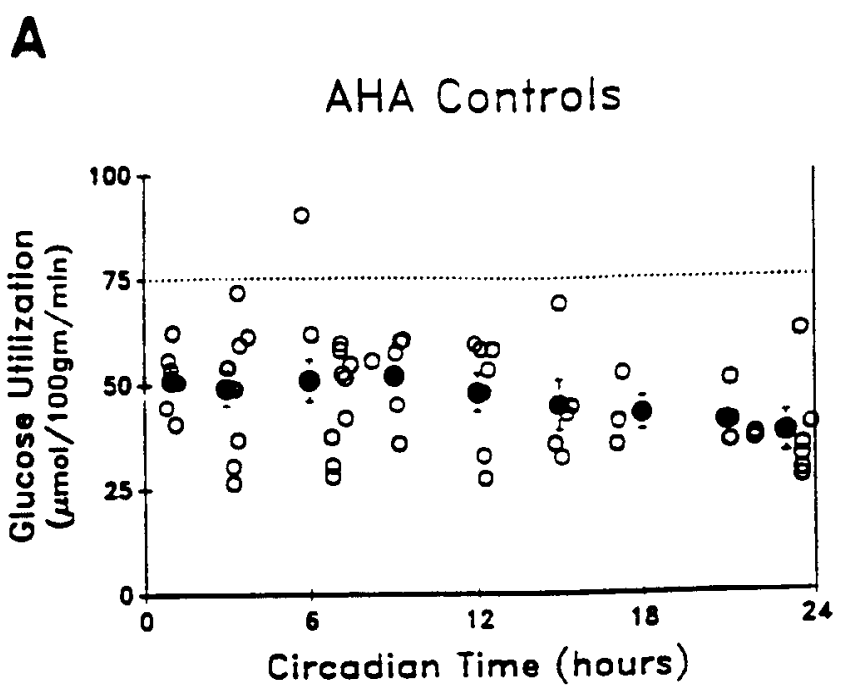

$\mathbf{B}$

\section{AHA with TTX}

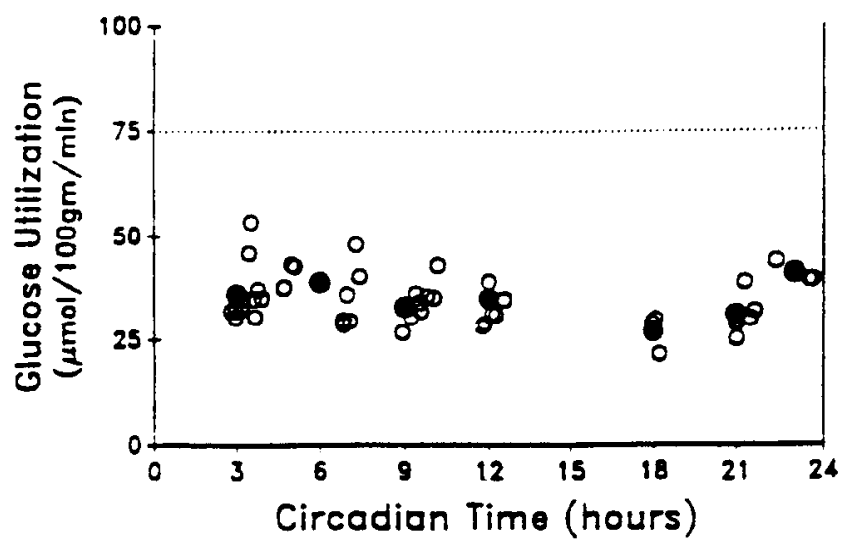

Figure 3. A, AHA glucose utilization of slices incubated under control conditions. $B$, AHA glucose utilization of slices incubated in the presence of TTX. Open circles denote individual values of AHA glucose utilization at exact circadian times, and solid circles refer to means and SEs of slices grouped by circadian times.

Table 1. Effects of $\mathrm{Mg}^{2+}$ on SCN glucose utilization at CT06

\begin{tabular}{clll}
$\begin{array}{c}\mathrm{Mg}^{2+} \\
(\mathrm{mM})\end{array}$ & $\mathrm{SCN}^{a}$ & $\mathrm{AHA}^{a}$ & $n$ \\
\hline 0.0 & $100.0 \pm 24.5$ & $40.4 \pm 5.1$ & 2 \\
0.8 & $197.0 \pm 36.9$ & $43.3 \pm 3.5$ & 2 \\
1.3 & $190.0 \pm 39.0$ & $46.4 \pm 4.2$ & 4 \\
1.8 & $118.5 \pm 61.0$ & $40.0 \pm 4.0$ & 3 \\
5 & $125.1 \pm 38.6$ & $49.7 \pm 3.1$ & 3 \\
10 & $34.9 \pm 4.5$ & $40.7 \pm 1.6$ & 3 \\
20 & $58.2 \pm 10.7$ & $48.8 \pm 5.2$ & 3
\end{tabular}

$\overline{{ }^{a} \text { Means and SD of glucose utilization }(\mu \mathrm{mol} / 100 \mathrm{gm} / \mathrm{min}) \text { measured at indicated }}$ $\mathrm{Mg}^{2+}$ concentrations. SCN values are measured by image analysis of autoradiographs, and AHA values are from whole slice radioactivity after removal of the optic chiasm and anterior commissure. All slices were incubated between CTO5 and CT07.
Correlation of Area and Glucose Utilization

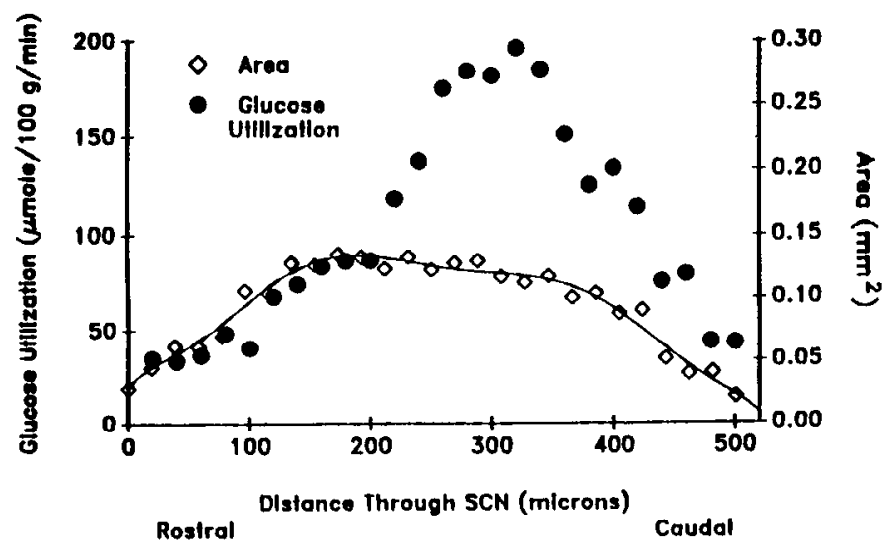

Figure 4. Correlation of cross-sectional area (open diamonds) and in vitro glucose utilization (solid circles) throughout the rostrocaudal extent of the SCN. Glucose utilization values are means of right and left SCN for three control slices, one each from CT06, CT09, and CT12. Area measurements represent the means of the six half-nuclei from the three slices. SE of glucose utilization measurements at each distance through the SCN averages $7.6 \mu \mathrm{mol} / 100 \mathrm{gm} / \mathrm{min}$, while area measurement SDs average $0.005 \mathrm{~mm}^{2}$. All three slices were aligned by using the most rostral section with a unilateral cross-sectional SCN area of $0.1 \mathrm{~mm}^{2}$ as a reference. The solid line represents the least-squares fit of an eighth order polynomial equation and is included to help illustrate the area profile of the SCN

$p<0.005)$. TTX does not significantly reduce AHA glucose utilization during the dark phase so that the AHA diurnal rhythm present under control conditions is not apparent in the presence of TTX. Notably, unlike the results observed for SCN, AHA glucose utilization of slices incubated at CT03 in the presence of TTX does not differ from AHA glucose utilization of slices incubated at other times of subjective daytime $(36.0 \pm 6.6$ and $35.9 \pm 6.3$ ). In the presence of TTX, SCN glucose utilization remains significantly above $\mathrm{AHA}$ glucose utilization during the dark phase of the L/D cycle (CT18 and CT21) with values of $49.9 \pm 9.8$ and $29.4 \pm 4.5$, respectively $(p<0.001)$.

\section{Effects of $M g^{2}$ ' on glucose utilization at CT06}

The effect of $\mathrm{Mg}^{2+}$ on $\mathrm{SCN}$ in vitro glucose utilization was studied in the presence of physiological concentrations of $\mathrm{Ca}^{2+}$ at a single circadian time (CT06) over a wide range of $\mathrm{Mg}^{2+}$ concentrations (Table 1). SCN glucose utilization is maximal at physiological concentrations of $\mathrm{Mg}^{2+}$ and dramatically reduced at 10 and 20 $\mathrm{mM}$, while AHA glucose utilization is virtually independent of $\mathrm{Mg}^{2+}$ concentration.

\section{Discussion}

The SCN offers a unique opportunity to study cerebral metabolism in vitro because it expresses intrinsic rhythms of neuronal and metabolic activity. Three components of in vitro SCN glucose utilization are identified by our results. The largest component is expressed during subjective daytime, correlates closely with a high neural firing rate (Green and Gillette, 1982; Groos and Hendriks, 1982; Shibata et al., 1982; Wheal and Thomson, 1984), and is suppressed by TTX. These features are consistent with the hypothesis that this daytime component of glucose utilization reflects primarily the metabolic costs of reestablishing ionic gradients in activated neural tissue (Mata et al., 1980; Sokoloff, 1982; Yarowsky et al., 1983). The second component 
consists of the SCN glucose utilization observed during the subjective dark phase. This low level of glucose utilization correlates with a low neural firing rate and is not significantly affected by TTX. Thus, it appears to represent basal metabolic activity related to cell maintenance and low levels of neural activity and neurotransmission. However, even at night, SCN glucose utilization remains above that of AHA, indicating a higher level of metabolic activity in these pacemaker neurons. The third component of SCN glucose utilization is observed at CT03 as metabolic activity that is above the basal level of nighttime SCN, or AHA, but that is not suppressible with TTX. Other studies have shown that $1 \mu \mathrm{M}$ TTX abolishes all sodium-dependent action potentials as well as optic nerve-evoked responses in SCN in vitro (Shibata et al., 1984a; Sugimori et al., 1984; S. Shibata and R. Y. Moore, unpublished observations). As might be expected from these data, TTX suppresses the in vitro rhythm of SCN VP release (Earnest et al., 1991). TTX, at concentrations of $0.3 \mu \mathrm{M}$, suppresses VP release and disrupts the subsequent circadian variation in VP release if given for 6 hr in late subjective day but not if given during early subjective day or for $12 \mathrm{hr}$ during subjective night. However, drinking rhythms appear to require at least three cycles for return of that rhythm after infusion of TTX into the region of SCN in vivo (Schwartz et al., 1987a), and there is some evidence of an underlying rhythm of VP release during the last days of explant culture following TTX exposure (Earnest et al., 1991). Although the nature of the TTX-resistant energy consuming processes at CT03 and whether they directly relate to oscillator function are presently unknown, the apparent rate of glucose consumption, at $30 \mu \mathrm{mol} / 100 \mathrm{gm} / \mathrm{min}$ above baseline, is considerable.

The primary difference between in vitro and in vivo SCN glucose utilization is the increased metabolic activity found in vitro during late subjective day. Examination of the in vitro rostrocaudal profile of SCN glucose utilization reveals that, unlike in vivo glucose utilization, which correlates with SCN crosssectional area throughout its rostrocaudal extent (Schwartz et al., 1987b), in vitro SCN glucose utilization correlates with crosssectional area only in the rostral half of the nucleus and is significantly increased in the caudal half relative to the rostral half and in vivo values. We suggest that two factors, anaerobic glycolysis and neural disinhibition, are sufficient to explain this discrepancy. It seems unlikely that the high SCN glucose utilization is related solely to in vitro radiotracer kinetics or methodology. That is, it should not represent some form of calculational error, since the values from rostral SCN and AHA are similar to those observed for SCN and hypothalamus in vivo (Schwartz et al., 1980; Schmidt et al., 1989), in vitro kinetic parameters for hypothalamus are similar to the analogous in vivo parameters, and the form of the rate equation employed for these studies is particularly insensitive to errors in the kinetic rate constants (Newman et al., 1990; Newman, 1991). Furthermore, glucose utilization measurements with slices from other regions of brain do not show values as high as those found in caudal SCN during subjective daytime, even after anoxia or upon $\mathrm{K}^{+}$stimulation (Newman et al., 1989, 1991). Obviously, the 2DG method alone cannot distinguish glycolysis associated with oxidative metabolism from strictly anaerobic glycolysis that would require much higher levels of glucose utilization for generating ATP. It is therefore interesting to note that SCN staining for cytochrome oxidase demonstrates high levels of cytochrome oxidase in the rostral and middle portions of SCN but much lower levels in the caudal third (Murakami and Fuller,
1988). The rostrocaudal pattern of this oxidative enzyme is thus the inverse of in vitro glucose utilization, suggesting that increased glucose utilization in caudal SCN may reflect higher glycolytic capacity in that region. Since the higher in vitro glucose utilization was observed in caudal SCN even when that portion of the nucleus was adjacent to the slice surface, diffusion of $\mathrm{O}_{2}$ into the slice is unlikely to explain this pattern. Furthermore, by itself, this tendency toward increased glycolysis cannot explain why in vitro SCN metabolic rates in late subjective day are higher than at other times of day relative to in vivo rates. Our present working hypothesis is that the increased metabolic rate in vitro reflects the loss of an inhibitor of SCN that is active in late daytime in vivo and that is lost when the SCN is isolated as a brain slice. The proposed inhibitory feedback could occur through either neural connections or humoral influences in cerebrospinal fluid or blood. For example, interruption of the ascending serotonergic pathway from dorsal raphe in vivo has been shown to increase glucose utilization significantly in SCN (Hery et al., 1982; Maxwell and Fink, 1988). The absence of inhibitory neuropeptides or other messengers, such as melatonin (Cassone et al., 1988), may also contribute to the observed high levels of glucose utilization observed in vitro. This explanation is analogous to prior suggestions that the reduced metabolic rates of hippocampus observed in vitro are due to the loss of excitatory pathways during isolation (McIlwain and Bachelard, 1971; Lipton and Whittingham, 1984; Jurgensen and Wright, 1988; Newman et al., 1989). However, because the SCN intrinsically generates a reliable pattern of neural firing rate even as a surgical "island" in vivo (Inouye and Kawamura, 1979), the absence of neural input need not lead to reduced metabolic activity. Indeed, we are suggesting that the absence of inhibitory neural input to SCN in slices may result in increased metabolic rate.

High regional glucose utilization has frequently been correlated with high regional synaptic content (Kennedy et al., 1976; Schwartz et al., 1979; Kadekaro et al., 1987), yet direct confirmation of this association is lacking. We have studied SCN metabolic activity at various $\mathrm{Mg}^{2+}$ concentrations in an effort to address such issues. High levels of $\mathrm{Mg}^{2+}$ have been observed to block synaptic transmission by both presynaptic and postsynaptic mechanisms (Crunelli and Mayer, 1984; Garthwaite and Garthwaite, 1987; Mayer and Westbrook, 1987; Cotman et al., 1988). Despite this, mean spontaneous SCN neural firing rates appear to be only minimally affected by high $\mathrm{Mg}^{2+}$ when $\mathrm{Ca}^{2+}$ is maintained in the normal range (Shibata et al., 1984b; Thomson, 1984). The marked suppression of SCN glucose utilization in vitro by concentrations of $\mathrm{Mg}^{2+}$ known to block synaptic transmission, combined with the relative insensitivity of SCN neural firing to high $\mathrm{Mg}^{2+}$ with normal $\mathrm{Ca}^{2+}$, provides direct support for the hypothesis that physiologically activated glucose utilization correlates with synaptic activity rather than with action potentials in perikarya or axons. This interpretation is not necessarily in conflict with the observed circadian rhythm of 2DG uptake in fetal rats prior to synapse formation since we observe enhanced glucose utilization in SCN brain slices at CT03 even in the presence of TTX and, in the absence of fetal rat kinetic parameters, the amplitude of the fetal rhythm is uncertain (Reppert and Schwartz, 1983, 1984; Moore and Bernstein, 1989). In fact, developmental studies of brain glucose utilization in rats indicate that rates of glucose utilization at birth are well below those of the adult (Nehlig et al., 1988).

These studies using the new quantitative method of brain 
slice glucose utilization support previous reports that the $\mathrm{SCN}$ continues to express circadian rhythms when isolated in vitro. The spontaneous properties of the SCN provide a unique model system for studying neural physiology. Brain slice glucose utilization is complementary to electrophysiology because information can be obtained even in the absence of neural action potentials. Thus, we have used TTX to expose energy consuming cellular processes in SCN apparently unrelated to $\mathrm{Na}^{+}$-dependent action potentials in early day. The quantitative nature of in vitro glucose utilization also permits direct comparison to in vivo studies. Future studies with $2 \mathrm{DG}$ and a variety of other radiotracers should increase our understanding of circadian rhythmicity as well as of neuronal regulation.

\section{References}

Cassone VM, Roberts MH, Moore RY (1988) Effects of melatonin on 2-deoxy-[1-14 C]glucose uptake within rat suprachiasmatic nucleus. Am J Physiol 255:R332-R337.

Cotman CW, Monaghan DT, Ganong AH (1988) Excitatory amino acid neurotransmission. Annu Rev Neurosci 11:61-80.

Crunelli V, Mayer ML (1984) $\mathrm{Mg}^{2+}$ dependence of membrane resistance increases evoked by NMDA in hippocampal neurones. Brain Res 311:392-396.

Earnest DJ, Digiorgio SM, Sladek CD (1991) Effects of tetrodotoxin on the circadian pacemaker mechanism in suprachiasmatic explants in vitro. Brain Res Bull 26:677-682.

Garthwaite G, Garthwaite J (1987) Receptor-linked ionic channels mediate $N$-methyl-D-aspartate neurotoxicity in rat cerebellar slices. Neurosci Lett 83:241-246.

Gillette MU (1991) SCN electrophysiology in vitro rhythmic activity. In: Suprachiasmatic nucleus: the mind's clock (Klein D, Moore RY, Turek F, eds), pp 125-143. New York: Oxford UP.

Green DJ, Gillette R (1982) Circadian rhythm of firing rate recorded from single cells in the rat suprachiasmatic brain slice. Brain Res 245: 198-200.

Groos G, Hendriks J (1982) Circadian rhythms in electrical discharge of rat suprachiasmatic neurones recorded in vitro. Neuroscience 34 : 283-288.

Hery M, Dusticier G, Calas A (1982) Application of the 2-deoxy-(1${ }^{14} \mathrm{C}$ ) glucose method to the study of suprachiasmatic nucleus activity and functions: phasic luteinizing hormone secretion and serotonin innervation. Exp Brain Res 47:465-468.

Inouye ST, Kawamura H (1979) Persistence of circadian rhythmicity in a mammalian hypothalamic "island" containing the suprachiasmatic nucleus. Proc Natl Acad Sci USA 76:5962-5966.

Jurgensen MB, Wright DC (1988) The effect of unilateral and bilateral removal of the entorhinal cortex on the glucose utilization in various hippocampal regions in the rat. Neurosci Lett 87:227-232.

Kadekaro M, Vance WH, Terrel ML, Gary H, Eisenberg HM, Sokoloff L (1987) Effects of antidromic stimulation of the ventral root on glucose utilization in the ventral horn of the spinal cord of the rat. Proc Natl Acad Sci USA 84:5492-5495.

Kennedy C, Des Rosiers MH, Sakurada O, Shinohara M, Reivich M, Jehle JW, Sokoloff L (1976) Metabolic mapping of the primary visual system of the monkey by means of autoradiographic $\left[{ }^{14} \mathrm{C}\right]$ technique. Proc Natl Acad Sci USA 73:4230-4234.

Lipton P, Whittingham TS (1984) Energy metabolism and brain slice function. In: Brain slices (Dingledine R, ed), pp 113-154. New York: Plenum.

Mata M, Fink DJ, Gainer H, Smith CB, Davidsen L, Savaki H, Schwartz WJ, Sokoloff L (1980) Activity-dependent energy metabolism in rat posterior pituitary primarily reflects sodium pump activity. J Neurochem 34:213-215.

Maxwell RC, Fink G (1988) The connections between the suprachiasmatic, ventrolateral geniculate and raphe nuclei studied by uptake of $\left[{ }^{14} \mathrm{C}\right] 2$-deoxyglucose. Neuroscience 24:265-274.

Mayer ML, Westbrook GL (1987) $\mathrm{Ca}^{++}$permeation through NMDA receptor channels. J Physiol (Lond) 394:501-527.

Mcllwain H, Bachelard HS (1971) Biochemistry and the central nervous system. Edinburgh: Churchill.
Meijer JH, Rietveld WJ (1989) Neurophysiology of the suprachiasmatic circadian pacemaker in rodents. Physiol Rev 69:671-707.

Moore RY, Bernstein ME (1989) Synaptogenesis in the rat suprachiasmatic nucleus demonstrated by electron microscopy and synapsin I immunoreactivity. J Neurosci 9:2151-2162.

Moore RY, Eichler VB (1972) Loss of a circadian adrenal corticosterone rhythm following suprachiasmatic lesions in the rat. Brain Res 42:201-206.

Murakami DM, Fuller CA (1988) The postnatal development of oxidative metabolism in the suprachiasmatic nucleus of the rat. Neuroscience 24:977-986.

Nehlig A, de Vasconcelos AP, Boyet S (1988) Quantitative autoradiographic measurement of local cerebral glucose utilization in freely moving rats during postnatal development. J Neurosci 8:2321-2333.

Newman GC (1991) SCN metabolic activity in vitro. In: Suprachiasmatic nucleus: the mind's clock (Klein D, Moore RY, Turek F, eds), pp 157-176. New York: Oxford UP.

Newman GC, Hospod FE (1986) Rhythm of suprachiasmatic nucleus 2-deoxyglucose uptake in vitro. Brain Res 381:345-350.

Newman GC, Hospod FE, Wu P (1989) Glucose utilization of ischcmic hippocampal slices. J Neurosci Methods 28:23-34.

Newman GC, Hospod FE, Patlak CS (1990) Kinetic model of 2-deoxyglucose metabolism using brain slices. J Cereb Blood Flow Metab 10:510-526.

Newman GC, Schissel S, Hospod FE (1991) Effects of $\mathrm{K}^{+}$and $\mathrm{pH}$ on ischemic brain slices. J Cereb Blood Flow Metab 11:398-406.

Reppert S, Schwartz WJ (1983) Maternal coordination of the fetal biological clock in utero. Science 220:969-971.

Reppert S, Schwartz WJ (1984) The suprachiasmatic nuclei of the fetal rat: characterization of a functional circadian clock using ${ }^{14} \mathrm{C}$-labeled deoxyglucose. J Neurosci 4:1677-1682.

Reppert SM, Uhl GR (1987) Vasopressin messenger ribonucleic acid in supraoptic and suprachiasmatic nuclei: appearance and circadian regulation during development. Endocrinology 120:2483-2487.

Robinson BG, Frim DM, Schwartz WJ, Maizoub JA (1988) Vasopressin mRNA in the suprachiasmatic nuclei: daily regulation of polyadenylate tail length. Science 241:342-344.

Schmidt K, Lucignani G, Mori K, Jay T, Palombo E, Nelson T, Pettigrew K, Holden JE, Sokoloff L (1989) Refinement of the kinetic model of the $2-\left[{ }^{14} \mathrm{C}\right]$ deoxyglucose method to incorporate effects of intracellular compartmentation in brain. J Cereb Blood Flow Metab 9:290-303.

Schwartz WJ, Reppert SM (1985) Neural regulation of the circadian vasopressin rhythm in cerebrospinal fluid: a pre-eminent role for the suprachiasmatic nuclei. J Neurosci 5:2771-2778.

Schwartz WJ, Smith CB, Davidsen L, Savaki H, Sokoloff L, Mata M, Fink D, Gainer H (1979) Metabolic mapping of functional activity in the hypothalamo-neurohypophysial system of the rat. Science 205: 723-725.

Schwartz WJ, Davidsen IC, Smith CB (1980) In vivo metabolic activity of a putative circadian oscillator, the rat suprachiasmatic nucleus. $\mathbf{J}$ Comp Neurol 189:157-167.

Schwartz WJ, Gross RA, Morton MT (1987a) The suprachiasmatic nuclei contain a tetrodotoxin-resistant circadian pacemaker. Proc Natl Acad Sci USA 84:1694-1698.

Schwartz WJ, Lydic R, Moore-Ede MC (1987b) In vivo metabolic activity of the suprachiasmatic nuclei: non-uniform intranuclear distribution of ${ }^{14} \mathrm{C}$-labeled deoxyglucose uptake. Brain Res 424:249-257.

Shibata S, Oomura Y, Kita H, Hattori K (1982) Circadian rhythmic changes of neuronal activity in the suprachiasmatic nucleus of the rat hypothalamic slice. Brain Res 247:154-158.

Shibata S, Oomura Y, Hattori K, Kita K (1984a) Responses of suprachiasmatic nucleus neurons to oplic nerve stimulation in rat hypothalamic slice preparation. Brain Res 302:83-89.

Shibata S, Shiratsuchi A, Liou SY, Ueki S (1984b) The role of calcium ions in circadian rhythm of suprachiasmatic nucleus neuron activity in rat hypothalamic slices. Neurosci Lett 52:181-184.

Sokoloff L (1982) The radioactive deoxyglucose method: theory, procedure and applications for the measurement of local glucose utilization in the central nervous system. Adv Neurochem 4:1-82.

Sokoloff L, Reivich M, Kennedy C, Des Rosiers M, Patlak CS, Pettigrew $\mathrm{KD}$, Sakurada O, Shinohara M (1977) The $\left[{ }^{14} \mathrm{C}\right]$ deoxyglucose method for the measurement of local cerebral glucose utilization. Theory, procedure and normal values in the conscious and anesthetized albino rat. J Neurochem 28:879-916. 
Stephan FK, Zucker I (1972) Circadian rhythms in drinking behavior and locomotor activity of rats are eliminated by hypothalamic lesions. Proc Natl Acad Sci USA 69:1583-1586.

Sugimori M, Shibata S, Oomura Y (1984) Electrophysiological basis for biorhythmic activity in the suprachiasmatic nucleus of the rat: an in vitro study. Soc Neurosci Abstr 10:294.

Thomson AM (1984) Slow, regular discharge in suprachiasmatic neurones is calcium dependent, in slices of rat brain. Neuroscience 13: 761-767.
Wheal HV, Thomson AM (1984) The electrical properties of neurones of the rat suprachiasmatic nucleus recorded intracellularly in vitro. Neuroscience 13:97-104.

Yarowsky P, Kadekaro M, Sokoloff L (1983) Frequency-dependent activation of glucose utilization in the superior cervical ganglion by electrical stimulation of cervical sympathetic trunk. Proc Natl Acad Sci USA 80:4179-4183. 